\title{
Evidence for a Type-II band alignment between cubic and hexagonal phases of GaN
}

\author{
X. H. Lu and P. Y. Yu ${ }^{\text {a) }}$ \\ Physics Department, University of California-Berkeley, Berkeley, California 94720 \\ and Materials Sciences Division, Lawrence Berkeley National Laboratory, Berkeley, California 94720 \\ L. X. Zheng, S. J. Xu, M. H. Xie, and S. Y. Tong ${ }^{\text {b) }}$ \\ Physics Department, The University of Hong Kong, Hong Kong
}

(Received 12 September 2002; accepted 3 December 2002)

\begin{abstract}
The photoluminescence spectra of a series of thin, undoped, hexagonal GaN films containing cubic $\mathrm{GaN}$ inclusions grown by molecular-beam epitaxy on $6 \mathrm{H}-\mathrm{SiC}$ have been studied as a function of temperature and excitation power. The dependence of the line shape and peak position of a peak at $\sim 3.17 \mathrm{eV}$ on laser power suggests that it is associated with a spatially indirect Type-II transition between hexagonal and cubic GaN. The values of the band offsets extracted from our data are in good agreement with theoretical predictions. (c) 2003 American Institute of Physics.
\end{abstract}

[DOI: $10.1063 / 1.1541113$ ]

When two semiconductors with different band gaps form a heterojunction, their bands can align with each other in at least three different ways. ${ }^{1}$ In the so-called Type-II alignment, the conduction band minimum and the valence band maximum occur in different semiconductors. As a result, photoexcited electrons and holes are spatially separated and their emission spectra exhibit unusual dependence on excitation power, such as a blueshift of the emission peak rather than a redshift, as expected from heating. ${ }^{2}$ Theory has predicted a Type-II band alignment between wurtzite $\mathrm{GaN}$ (w$\mathrm{GaN}$, with band gap $E_{g} \sim 3.47 \mathrm{eV}$ ) and zincblende $\mathrm{GaN}$ (z$\mathrm{GaN}$, with $E_{g} \sim 3.26 \mathrm{eV}$ ), with the conduction band minimum in z-GaN and the valence band maximum in w-GaN. ${ }^{3,4}$ The predicted value of the conduction band offset is $\sim 0.25 \mathrm{eV}$, while the photon energy of the spatially indirect transition is $\sim 3.20 \mathrm{eV}$.

These predictions have not yet been verified experimentally because it is not possible to grow a controlled heterojunction between $\mathrm{w}-\mathrm{GaN}$ and $\mathrm{z}-\mathrm{GaN}$. The stable crystal structure of $\mathrm{GaN}$ is wurtzite, while $\mathrm{z}-\mathrm{GaN}$ has been grown only as a thin film on cubic substrates like GaAs and $3 \mathrm{C}-\mathrm{SiC} .{ }^{5}$ There is, however, evidence suggesting that inclusions of z-GaN can exist in w-GaN as stacking faults, and vice versa. For example, w-GaN in z-GaN films has been detected both by photoluminescence (PL) and Raman scattering. ${ }^{5}$ Similarly, z-GaN is often found in w-GaN films by high-resolution transmission electron microscopy (HRTEM). Some below-band-gap emission peaks in PL spectra of $\mathrm{w}-\mathrm{GaN}$ have been attributed to stacking faults. ${ }^{6,7}$ However, no experimental evidence indicates that these stacking fault emissions behave like spatially indirect transitions. In this letter, we report a study of the PL spectra of a series of thin $\mathrm{w}-\mathrm{GaN}$ films whose HRTEM shows the presence of z-GaN inclusions. In the PL spectra of one sample, we found evidence of a spatially indirect transition at $\sim 3.17 \mathrm{eV}$ which cannot be attributed to a donor-acceptor pair transition.

\footnotetext{
${ }^{a)}$ Electronic mail address: pyyu@1bl.gov

${ }^{b)}$ Present address: City University of Hong Kong, Hong Kong.
}

From an analysis of our results based on a Type-II band alignment, we deduced a value of $\sim 0.3 \mathrm{eV}$ for the conduction band offset between w-GaN and z-GaN, in good agreement with theoretical predictions.

Our w-GaN thin films were grown by molecular-beam epitaxy on $6 \mathrm{H}-\mathrm{SiC}$ substrates. Details of the growth have been described elsewhere ${ }^{8}$ and will not be repeated here. We have studied a series of films varying in thickness from $\sim 5$ $\mathrm{nm}$ to $\sim 3 \mu \mathrm{m}$. The samples, mounted in a variabletemperature He closed-cycle refrigerator, were excited by the 3.532- and 3.408-eV lines of an Ar-ion laser. The former laser will excite both $\mathrm{w}-\mathrm{GaN}$ and $\mathrm{z}-\mathrm{GaN}$, while latter will excite only z-GaN. The importance of using the $3.408-\mathrm{eV}$ line is that its energy is high enough to excite any possible Type-II transitions. The emission spectra were analyzed with a Spex triple spectrometer equipped with a cooled CCD detector. The spectral resolution of the system was $\sim 1 \mathrm{meV}$.

Among our samples, we found that only one $\mathrm{GaN}$ sample (M083) has an emission peak at $\sim 3.17 \mathrm{eV}$ whose power dependence is consistent with a spatially indirect transition. This is not surprising, since we expect Type-II heterojunctions to be formed only rarely, when a large region of $\mathrm{z}-\mathrm{GaN}$ occurs in w-GaN. Figure 1 shows a series of PL spectra obtained from sample M083 $(\sim 120 \mathrm{~nm}$ thick) as a function of sample temperature $(T)$ when excited by a relatively low laser power of $23 \mathrm{~mW}$ (focused to a spot $\sim 100 \mu \mathrm{m}$ in size). The sharp peaks in this figure are due to Raman scattering from both the $\mathrm{w}-\mathrm{GaN}$ film and the $6 \mathrm{H}-\mathrm{SiC}$ substrate and are not of interest in this letter. The rising tail at energies below $3.10 \mathrm{eV}$ represents emission from the $\mathrm{SiC}$ substrate. We shall concentrate on the two broad and strong peaks at 3.17 and $3.26 \mathrm{eV}$. The latter peak is present in nearly all of our samples except for the thickest film. Because its energy is very close to the $E_{g}$ of $\mathrm{z}-\mathrm{GaN},{ }^{5}$ we attribute it to gap emission from $\mathrm{z}-\mathrm{GaN}$ inclusions in our samples. As $T$ is increased from 14 to $80 \mathrm{~K}$, both the 3.17 and $3.26 \mathrm{eV}$ emission peaks exhibit a small redshift, some broadening and thermal quenching of peak intensity. By deconvolving the PL spectra into a sum of Gaussian peaks, we obtained Arrhenius plots of 


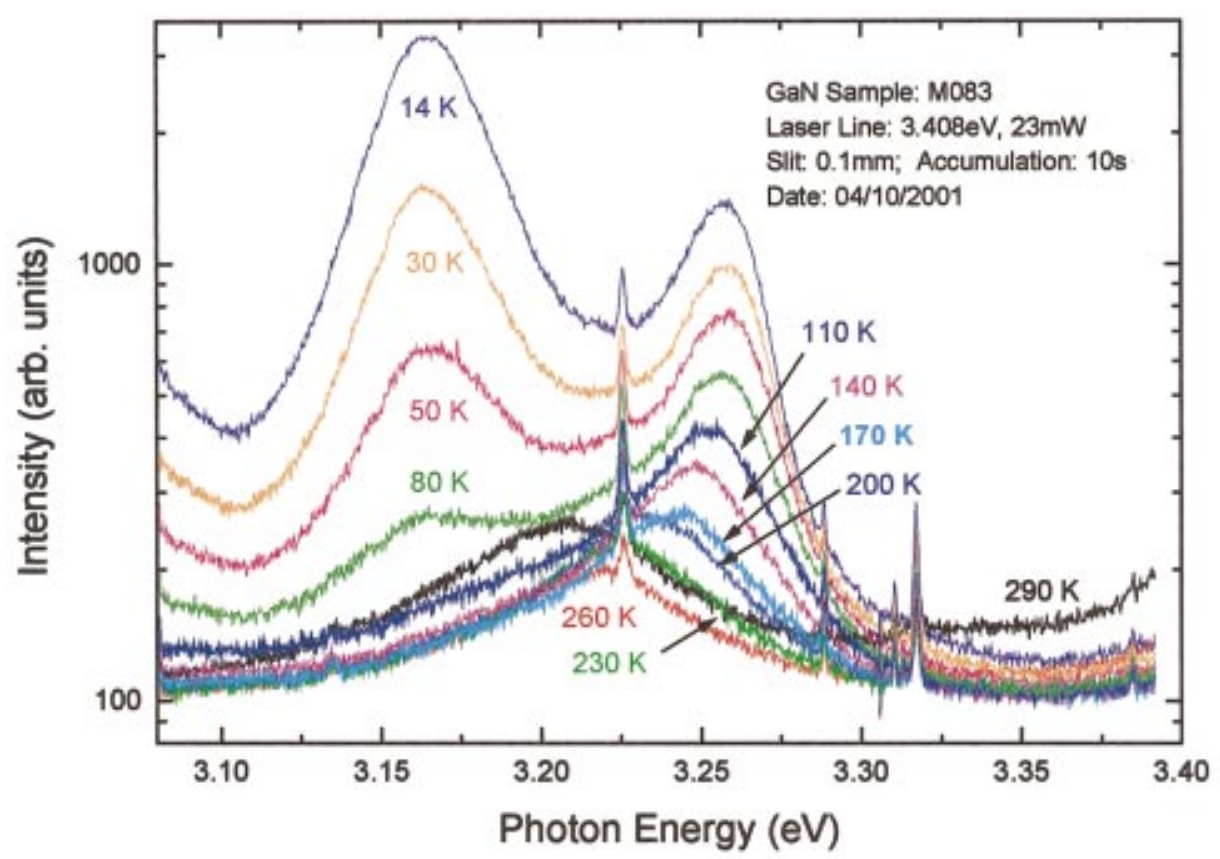

FIG. 1. (Color) The emission spectra of GaN/6H-SiC sample M083 excited by the 3.408-eV line of the Ar-ion laser measured at various temperatures.

the 3.17- and 3.26-eV peaks and determined their activation energies for thermal quenching as 11 and $29 \mathrm{meV}$, respectively. The activation energy of the $3.26-\mathrm{eV}$ peak is consistent with the identification of this peak as due to either a free exciton or a exciton bound to a shallow donor. However, the thermal quenching of the $3.17-\mathrm{eV}$ peak is unusually strong. Its activation energy is too small even for shallow donors in $\mathrm{GaN!}$

We next excite sample M083 at $3.408 \mathrm{eV}$ with laser powers $(P)$ up to $400 \mathrm{~mW}$, while keeping the size of the focal spot constant. The low-temperature emission spectra mea- sured at various values of $P$ are shown in Fig. 2. The striking features of these spectra are the decrease in peak intensity and a blueshift in peak position of the $3.17 \mathrm{eV}$ peak at high $P$. A weaker saturation effect is also found in the $3.26-\mathrm{eV}$ peak at $P>250 \mathrm{~mW}$, probably as a result of laser-induced heating. While the $3.26-\mathrm{eV}$ peak can be fitted with a Gaussian at both high and low values of $P$, there are deviations of the 3.17-eV peak line shape from a Gaussian at high $P$. This is shown more clearly in Fig. 3, where the 3.26-eV peak has been subtracted off from the experimental spectra (open circles). The resultant peak (solid circles) shows a maximum

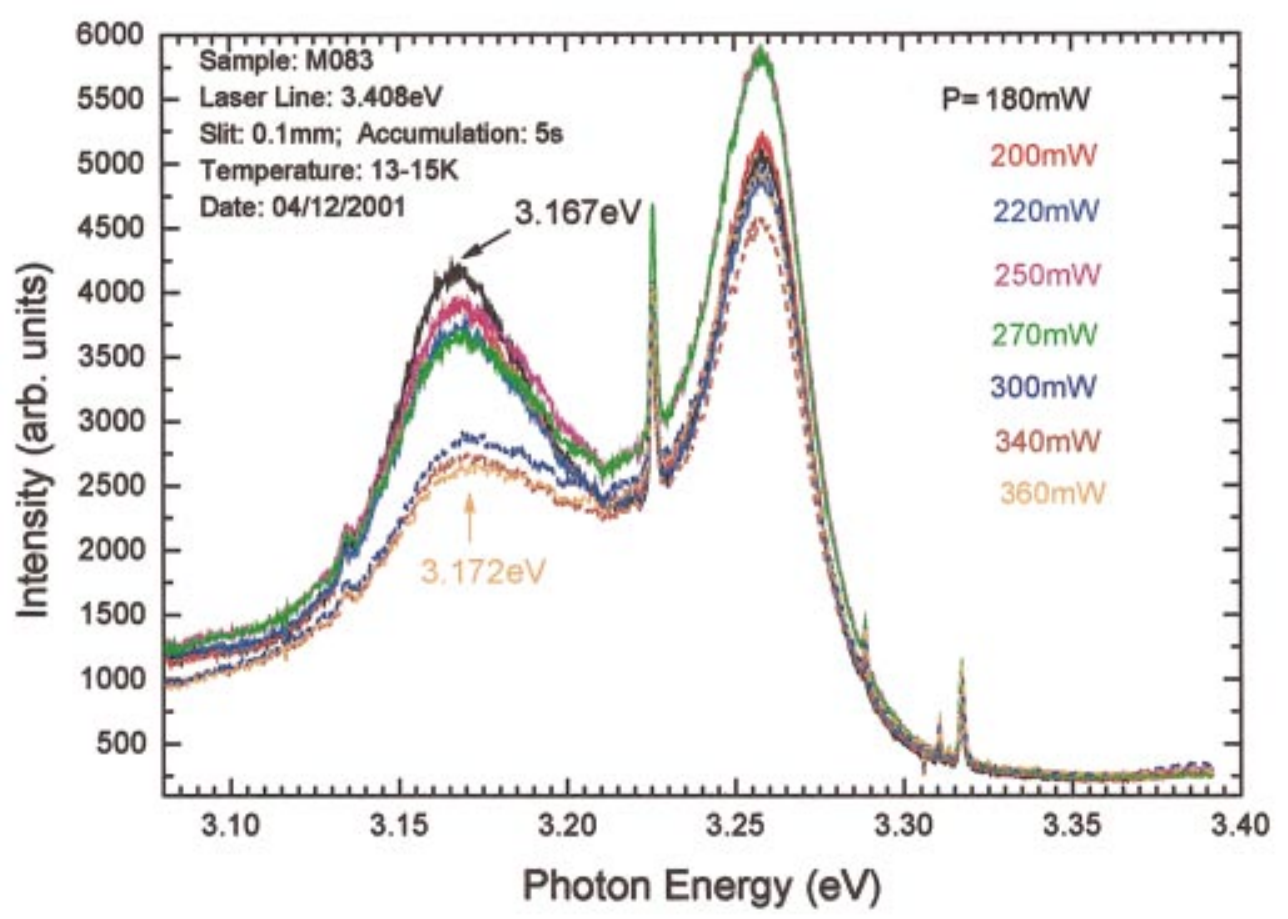

FIG. 2. (Color) Low-temperature emission spectra of sample M083 excited by the 3.408-eV line of the Ar-ion laser measured at various excitation laser powers $P$. 


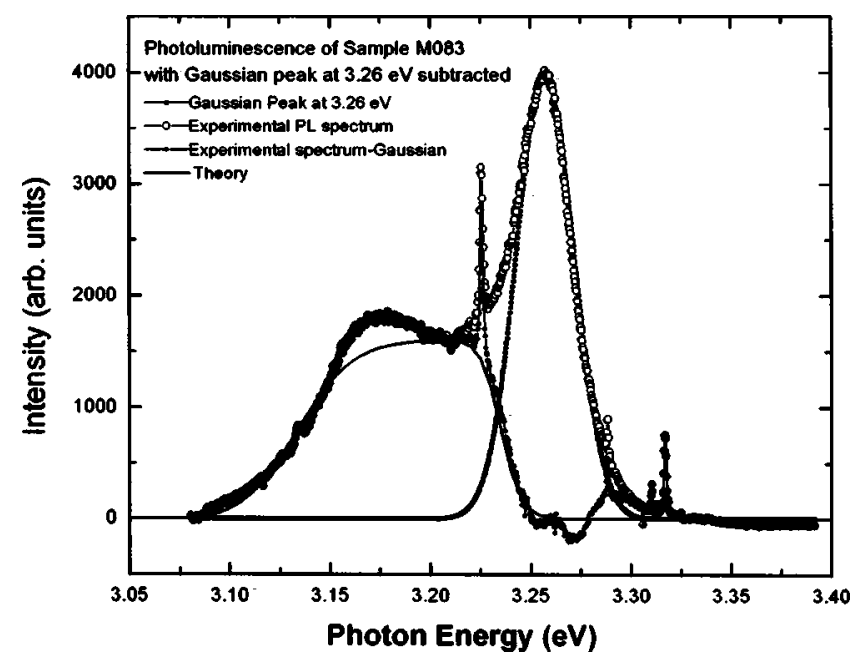

FIG. 3. Decomposition of the high-intensity excitation emission spectrum (open circles) of sample M083 into the sum of a Gaussian peak at $3.26 \mathrm{eV}$ (closed circles) and a asymmetrical peak around $3.17 \mathrm{eV}$ (crosses). The solid curve is a plot of the theoretical expression [Eq. (1)] using the fitting parameters $T=40 \mathrm{~K}, \gamma=29 \mathrm{meV}, \mu_{\mathrm{e}}=105 \mathrm{meV}$, and $E_{i g}=3.13 \mathrm{eV}$.

at $3.172 \mathrm{eV}$ and a sharp cutoff on the high-energy side. The dependence of the 3.17-eV peak line shape on $P$ suggests that it may be a spatially indirect transition associated with a Type-II band alignment.

To test this idea further, we have analyzed its line shape under high intensity excitation. We assume that the photoexcited electron and holes are separated by the electric field present at the Type-II band alignment to form degenerate, two-dimensional plasmas with quasi-Fermi levels $\mu_{\mathrm{e}}$ and $\mu_{\mathrm{h}}$. For simplicity, we shall assume that only one of the valence bands in $\mathrm{GaN}$ is populated, and neglect any dependence of the optical transition matrix element $M$ on the emission energy. In this approximation, the emission line shape $I\left(\omega_{\mathrm{em}}\right)$ is mainly dependent on the electron and hole densityof-states $D_{\mathrm{e}}\left(E_{\mathrm{e}}\right)$ and $D_{\mathrm{h}}\left(E_{\mathrm{h}}\right)$ (which are step functions) and the spatially indirect band gap $E_{i g}$ :

$$
\begin{aligned}
I\left(\omega_{\mathrm{em}}\right) \sim & \iint D_{\mathrm{e}}\left(E_{\mathrm{e}}\right) D_{\mathrm{h}}\left(E_{\mathrm{h}}\right) f_{\mathrm{e}}\left(E_{\mathrm{e}}\right) f_{\mathrm{h}}\left(E_{\mathrm{h}}\right) \\
& \times \delta E_{i g}\left(E_{\mathrm{e}}+E_{\mathrm{h}}-\hbar \omega_{\mathrm{em}}\right) d\left(E_{\mathrm{e}}\right) d\left(E_{\mathrm{h}}\right) .
\end{aligned}
$$

In Eq. (1), $f_{\mathrm{e}}$ and $f_{\mathrm{h}}$ are, respectively, the electron and hole distribution functions. Since the hole mass is about three times larger than the electron mass in $\mathrm{GaN},{ }^{9}$ we shall neglect the dependence of $\mu_{\mathrm{h}}$ on the hole density when compared to the dependence of $\mu_{\mathrm{e}}$ on the electron density. To explain the low-energy tail in the emission peak, we broaden the densityof-states with a Gaussian characterized by a damping constant $\gamma$. The resultant expression used to fit the experimental data has four adjustable parameters: $T, \gamma, \mu_{\mathrm{e}}$, and $E_{i g}$. These parameters can be determined independently from the experimental curve in Fig. 3 since they affect the emission line shape differently. The solid curve in Fig. 3 represents the best fit to the data and is obtained with the following parameters: $T=40 \mathrm{~K}, \quad \gamma=29 \mathrm{meV}, \quad \mu_{\mathrm{e}}=105 \mathrm{meV}, \quad$ and $E_{i g}$
$=3.13 \mathrm{eV}$. The slight peaking of the experimental spectrum at $3.17 \mathrm{eV}$ can be explained by the fact that the transition matrix element $M$ is not constant, but should depend very strongly on the spatial overlap of the electron and hole wave functions. We expect $M$ to be larger for emissions near the band gap because the lower-energy electrons and holes are more localized near the interface.

From the values of the spatially indirect energy gap $E_{g}$ deduced from our measurement, we determined the conduction band offset between w-GaN and z-GaN to be $0.3 \mathrm{eV}$ by neglecting effects of band bending and band-gap renormalization. This value is in good agreement with the theoretically predicted value of $0.25 \mathrm{eV},{ }^{4}$ considering the experimental uncertainties. The small valence band offset of $\sim 90 \mathrm{meV}$ suggests that the holes are only weakly bound in w-GaN. This small binding energy of the holes accounts for the unusually small activation energy for thermal quenching of the spatially indirect transition. Indeed the intensity of the 3.26 $\mathrm{eV} \mathrm{c}-\mathrm{GaN}$ peak increases at $T>170 \mathrm{~K}$ after the 3.17-eV peak becomes completely quenched. The sudden drop in the intensity of the $3.17-\mathrm{eV}$ peak accompanied by a line shape change at $P>270 \mathrm{~mW}$ can be understood in terms of the dissociation of the indirect excitons into a degenerate twodimensional plasma.

In summary, we found that an emission peak at $\sim 3.17$ $\mathrm{eV}$ in thin, hexagonal GaN films grown on $6 \mathrm{H}-\mathrm{SiC}$ and containing cubic phase of $\mathrm{GaN}$ as inclusions exhibits behaviors typical of a spatially indirect transition. Its very small thermal activation energy suggests that it is not due to a donoracceptor transition. A quantitative fit of its emission line shape based on a Type-II transition yields a conduction band offset between wurtzite and cubic GaN, in good agreement with theory.

The work at Berkeley was supported by the US Department of Energy under Contract No. DE-AC03-76SF00098, while the work at HKU was supported by the HK Research Grants Council grant No. HKU 7121/00P and by the NFSC/ RGC joint research grant No. N-CityU028/00.

\footnotetext{
${ }^{1}$ See, for example, P. Y. Yu and M. Cardona, Fundamentals of Semiconductors: Physics and Materials Properties, 3rd ed. (Springer, Berlin, 2001), p. 479.

${ }^{2}$ Such an intensity-dependent blueshift has also been observed in donoracceptor pair emission in GaN. See J. S. Colton and P. Y. Yu, Appl. Phys. Lett. 78, 2500 (2001), and references therein.

${ }^{3}$ Z. Z. Bandić, T. C. McGill, and Z. Ikonic, Phys. Rev. B 56, 3564 (1997).

${ }^{4}$ C. Stampfl and C. G. Van de Walle, Phys. Rev. B 57, R15052 (1998).

${ }^{5}$ B. Daudin, G. Feuillet, J. Hübner, Y. Samson, A. Widmann, A. Philippe, C. Bru-Chevallier, G. Guillot, E. Bustarret, G. Bentoumi, and A. Deneuville, J. Appl. Phys. 84, 2295 (1998).

${ }^{6}$ See, for example, Yihwan Kim, Ph.D. thesis, Materials Sciences Department, University of California, Berkeley, 2000.

${ }^{7}$ Y. T. Rebane, Y. G. Shreter, and M. Albrecht, Phys. Status Solidi A 164, 141 (1997).

${ }^{8}$ M. H. Xie, S. M. Seutter, W. K. Zhu, L. X. Zheng, Huasheng Wu, and S. Y. Tong, Phys. Rev. Lett. 82, 2749 (1999).

${ }^{9}$ See, for example, P. Y. Yu and M. Cardona, Fundamentals of Semiconductors: Physics and Materials Properties, 3rd ed. (Springer, Berlin, 2001), Tables 2.22 and 2.24.
} 